$09,04,05$

\title{
Обменные и обменно-релятивистские эффекты в возбужденных состояниях $3 d$-ионов в кристаллах
}

\author{
(C) A.C. Москвин
}

Уральский фредеральный университет,

Екатеринбург, Россия

E-mail: alexander.moskvin@urfu.ru

\begin{abstract}
Использование простейших спиновых гамильтонианов типа Гейзенберга или Дзялошинского-Мория, традиционных для основных орбитально-невырожденных состояний, не позволяет корректно описать особенности обменных и обменно-релятивистских взаимодействий для возбужденных состояний $3 d-$ и $4 f$-ионов в кристаллах. Нами рассмотрен обобщенный гамильтониан обменного и сверхобменного взаимодействий, который в рамках единого подхода позволяет учесть эффекты орбитального (квази)вырождения, обменный механизм переноса возбуждения. Рассмотрен новый механизм обменно-релятивистских взаимодействий „спин-чужая орбита“, в частности, спин-орбитальный аналог взаимодействия Дзялошинского-Мория и его проявление в циркулярной магнитооптике слабых ферромагнетиков.
\end{abstract}

Работа выполнена при поддержке Программы 211 Правительства Российской Федерации, соглашение № 02.А03.21.0006, и проектов № 2277 и № 5719 Министерства образования и науки Российской Федерации.

DOI: 10.21883/FTT.2019.05.47605.18F

\section{1. Введение}

Оптические и особенно магнитооптические свойства соединений на основе $3 d$ - и $4 f$-ионов определяются как свойствами оптически активного центра, так и его взаимодействиями с основными и возбужденными состояниями соседних ионов. В последние годы также активно изучаются оптически индуцированные магнитные фазовые превращения, связанные со специфическими магнитными свойствами и взаимодействиями для возбужденных состояний $3 d$-ионов в кристаллах. К сожалению, анализ обменных и обменно-релятивистских взаимодействий в возбужденных состояниях при этом чаще всего сводится к использованию спиновых гамильтонианов типа Гейзенберга или Дзялошинского-Мория, традиционных для основных орбитально-невырожденных состояний.

Такие подходы не позволяют учесть многие особенности возбужденных состояний, связанные прежде всего с наличием орбитального вырождения и существенным отличием численных значений, а иногда и знака, параметров взаимодействий от значений, типичных для основных состояний.

В данной работе мы рассмотрим наиболее общие операторные формы обменных и обменно-релятивистских взаимодействий, позволяющие дать адекватное описание соответствующих эффектов как для основных, так и для возбужденных состояний.

\section{2. Обменные взаимодействия в возбужденных состояниях 3d-ионов в кристаллах}

\section{1. Прямое обменное взаимодействие}

Наиболее общим, последовательным и эффективным методом описания обменного взаимодействия ионов с незаполненными $3 d$ - и $4 f$-оболочками является метод неприводимых тензорных операторов, или алгебра Рака, впервые использованный 50 лет назад в работе [1] (см. также работы [2-5]. Так, гамильтониан прямого обменного взаимодействия двух ионов с электронными конфигурациями $n_{1} l_{1}^{N_{1}}$ и $n_{2} l_{2}^{N_{2}}$ может быть представлен в виде

$$
\begin{aligned}
\hat{V}_{\mathrm{ex}}(1,2)= & \sum I^{*}\left(f_{1} l_{1} l_{1} f_{2} l_{2} l_{2} \mid b_{1} b_{2} b \beta\right) \\
& \times\left[\hat{W}^{a b_{1}}\left(l_{1} l_{1}\right) \times \hat{W}^{a b_{2}}\left(l_{2} l_{2}\right)\right]_{\beta}^{b},
\end{aligned}
$$

где

$$
\left[\hat{W}^{a b_{1}} \times \hat{W}^{a b_{2}}\right]_{\beta}^{b}=\sum_{\alpha \beta_{1} \beta_{2}}\left[\begin{array}{ccc}
b_{1} & b_{2} & b \\
\beta_{1} & \beta_{2} & \beta
\end{array}\right](-1)^{\alpha} \hat{W}_{\alpha \beta_{1}}^{a b_{1}} \hat{W}_{-\alpha \beta_{2}}^{a b_{2}}
$$

есть неприводимое тензорное произведение двойных тензорных операторов ранга $b$ в орбитальном пространстве и обычное скалярное произведение в спиновом пространстве, [:::] - коэффициенты Клебша-Гордана.

С учетом кинетического и потенциального вкладов обменные параметры могут быть представлены в виде:

$$
\begin{aligned}
& I\left(f_{1} l_{1} l_{1} f_{2} l_{2} l_{2} \mid b_{1} b_{2} b \beta\right)=\sum_{\beta_{1}, \beta_{2}} \sum_{m_{1}, m_{1}^{\prime}, m_{2}, m_{2}^{\prime}}\left[l_{1} l_{2}\right]^{\frac{1}{2}}\left[\begin{array}{ccc}
b_{1} & b_{2} & b \\
\beta_{1} & \beta_{2} & \beta
\end{array}\right] \\
& \times(-1)^{l_{1}-m_{1}}\left(\begin{array}{ccc}
l_{1} & b_{1} & l_{1} \\
-m_{1} & \beta_{1} & m_{1}^{\prime}
\end{array}\right)(-1)^{l_{2}-m_{2}}\left(\begin{array}{ccc}
l_{2} & b_{2} & l_{2} \\
-m_{2} & \beta_{2} & m_{2}^{\prime}
\end{array}\right) \\
& \times\left\{\frac{t\left(f_{1} l_{1} m_{1} \mid f_{2} l_{2} m_{2}^{\prime}\right) t\left(f_{2} l_{2} m_{2} \mid f_{1} l_{1} m_{1}^{\prime}\right)}{U}\right. \\
& \left.-\frac{1}{2} J\left(f_{1} l_{1} m_{1} f_{2} l_{2} m_{2} \mid f_{2} l_{2} m_{2}^{\prime} f_{1} l_{1} m_{1}^{\prime}\right)\right\}, \\
& \text { где } \quad \begin{array}{c}
U_{1} \\
J\left(f_{1} l_{1} m_{1} f_{2} l_{2} m_{2} \mid f_{2} l_{2} m_{2}^{\prime} f_{1} l_{1} m_{1}^{\prime}\right) \quad \text { - гнергия гейзенберговский }
\end{array}
\end{aligned}
$$


обменный интеграл, (:::) - коэффициент Вигнера. Пренебрежение межконфигурационными эффектами и смешиванием термов ${ }^{2 S+1} L$ приводит к четным значениям $\left(b_{1}+b_{2}\right)$ и $b$.

Несмотря на сложный вид обменных параметров, они имеют простую зависимость от направления радиусавектора пары $\mathbf{R}_{12}$ :

$$
I\left(f_{1} l_{1} l_{1} f_{2} l_{2} l_{2} \mid b_{1} b_{2} b \beta\right)=J\left(f_{1} l_{1} l_{1} f_{2} l_{2} l_{2} \mid b_{1} b_{2} b\right) C_{\beta}^{b}\left(\mathbf{R}_{12}\right),
$$

где обменные параметры $J\left(f_{1} l_{1} l_{1} f_{2} l_{2} l_{2} \mid b_{1} b_{2} b\right)$ зависят от $\left|\mathbf{R}_{12}\right|, C_{\beta}^{b}\left(\mathbf{R}_{12}\right)$ - сферическая тензорная гармоника.

Оператор обменного взаимодействия в неприводимой тензорной форме существенно отличается от простейшего оператора гайзенберговского обмена вида

$$
\hat{V}_{\mathrm{ex}}^{(H)}=2 I\left(\mathbf{S}_{1} \cdot \mathbf{S}_{2}\right)
$$

и может быть назван обобщенным гамильтонианом обменного взаимодействия. Этот оператор так же, как и его упрощенный вариант (4), сохраняет величину суммарного спина пары $\mathbf{S}_{12}=\mathbf{S}_{1}+\mathbf{S}_{2}$ и его проекцию. Однако в отличие от $\hat{V}_{e x}^{(H)}$ новый оператор может менять величину спина отдельного центра $S_{1}$ (или $S_{2}$ ), то есть фактически он может приводить к смешиванию различных спиновых состояний центра. Кроме того, обобщенный обменный гамильтониан является сложным орбитальным оператором с бесспиновой $(a=0)$ и спинзависимой $(a=1)$ частью. Тензорная структура бесспинового или чисто орбитального обмена существенно отличается от операторной структуры кулоновского взаимодействия, где $b=b_{1}+b_{2}$. Слагаемое $a=0$ соответствует бесспиновому, чисто орбитальному изотропному и анизотропному обмену. В частности, слагаемые с $b_{1}=b_{2}=1 ; b=0$ и $b_{1}=b_{2}=2 ; b=0$ сводятся к изотропному билинейному $\hat{V}_{11}=I_{11}\left(\hat{\mathbf{L}}_{1} \cdot \hat{\mathbf{L}}_{2}\right)$ и биквадратичному $\hat{V}_{22}=I_{22}\left(\hat{\mathbf{L}}_{1} \cdot \hat{\mathbf{L}}_{2}\right)^{2}$ орбитальному обмену, если учесть, что

$$
\begin{gathered}
{\left[\hat{W}_{f_{1}}^{01}\left(n_{1} l_{1}\right) \times \hat{W}_{f_{2}}^{01}\left(n_{2} l_{2}\right)\right]_{0}^{0}=\left(\hat{\mathbf{L}}_{1} \cdot \hat{\mathbf{L}}_{2}\right),} \\
{\left[\hat{W}_{f_{1}}^{02}\left(n_{1} l_{1}\right) \times \hat{W}_{f_{2}}^{02}\left(n_{2} l_{2}\right)\right]_{0}^{0}=\left(\hat{\mathbf{L}}_{1} \cdot \hat{\mathbf{L}}_{2}\right)+\left(\hat{\mathbf{L}}_{1} \cdot \hat{\mathbf{L}}_{2}\right)^{2} .}
\end{gathered}
$$

Слагаемые с $a=1$ дают спин-зависимый обмен, который при $b_{1}=b_{2}=b=0$ сводится к обычному гейзенберговскому обмену (4), но только при фиксированных значениях $L_{1} S_{1}$ и $L_{2} S_{2}$. При $b=0$ мы приходим к пространственно изотропной обменной связи.

Гамильтониан (1) включает недиагональные слагаемые с $L_{1} S_{1} \neq L_{1}^{\prime} S_{1}^{\prime}, L_{2} S_{2} \neq L_{2}^{\prime} S_{2}^{\prime}$, ответственные, в частности, за обменно-индуцированный перенос энергии [6], давыдовское расщепление и эффекты смешивания термов. Обменный гамильтониан (1) может быть легко обобщен на описание межконфигурационных эффектов $\left(n_{1} l_{1}-n_{1}^{\prime} l_{1}^{\prime}, \quad n_{2} l_{2}-n_{2}^{\prime} l_{2}^{\prime}\right)$, в частности, эффектов смешивания пространственной четности, имеющих важное значение для расчета спин-зависимой электрической поляризации и магнитоэлектрических эффектов [7].

\section{2. Сверхобменное взаимодействие}

Гамильтониан сверхобменного взаимодействия двух ионов с электронными конфигурациями $n_{1} l_{1}^{N_{1}}$ и $n_{2} l_{2}^{N_{2}}$ через промежуточный немагнитный ион - лиганд имеет то же самое выражение (1), что и для прямого обмена, однако со специфической зависимостью обменных параметров от геометрии сверхобмена $[4,5,8]$ :

$$
\begin{gathered}
I\left(f_{1} l_{1} l_{1} f_{2} l_{2} l_{2} \mid b_{1} b_{2} b \beta\right)=\sum_{k_{1} k_{2}} J\left(f_{1} l_{1} l_{1} f_{2} l_{2} l_{2} \mid b_{1} b_{2} k_{1} k_{2} b\right) \\
\times\left[C^{k_{1}}\left(\mathbf{R}_{10}\right) \times C^{k_{2}}\left(\mathbf{R}_{20}\right)\right]_{\beta}^{b} .
\end{gathered}
$$

Если мы пренебрегаем межконфигурационными эффектами для магнитных катионов, $\left(b_{1}+b_{2}\right)$ и $\left(k_{1}+k_{2}\right)-$ четные числа. Для сверхобменного взаимодействия, связанного с определенной $n_{0} l_{0}$-оболочкой лиганда, мы приходим к четным числам $k_{1}$ и $k_{2}$, подчиняющимся правилу треугольника: $k_{1,2} \leq 2 l_{0}$. Для механизма, связанного с межконфигурационным $n_{0} l_{0} \rightarrow n_{0}^{\prime} l_{0}^{\prime}$ возбуждением $\left|l_{0}-l_{0}^{\prime}\right| \leq k_{1,2} \leq\left(l_{0}+l_{0}^{\prime}\right)$, тогда как четность $k_{1,2}$ совпадает с четностью $\left(l_{0} \pm l_{0}^{\prime}\right)$. Кроме того,

$$
\begin{gathered}
\left|k_{1}-k_{2}\right| \leq b \leq k_{1}+k_{2}, \quad\left|b_{1,2}-c\right| \leq k_{1,2} \leq b_{1,2}+c, \\
\left|l_{0}-l_{0}^{\prime}\right| \leq c \leq l_{0}+l_{0}^{\prime},
\end{gathered}
$$

a $k_{1,2}+l_{0}+l_{0}^{\prime}, b_{1,2}+k_{1,2}+c, b_{1}+b_{2}$ являются четными числами.

При $b=0$ мы получаем пространственно изотропную сверхобменную связь с обменными параметрами, зависящими от геометрии сверхобмена следующим образом:

$$
\begin{aligned}
I\left(b_{1} b_{2} 00\right)=\sum_{k_{1} k_{2}}(-1)^{k_{1}}[ & \left.k_{1}\right]^{-1 / 2} \delta_{b_{1} b_{2}} \delta_{k_{1} k_{2}} \\
& \times J\left(b_{1} b_{2} k_{1} k_{2} 0\right) P_{k_{1}}\left(\cos \theta_{12}\right),
\end{aligned}
$$

так как

$$
\begin{gathered}
{\left[C^{k_{1}}\left(\mathbf{R}_{10}\right) \times C^{k_{2}}\left(\mathbf{R}_{20}\right)\right]_{0}^{0}=(-1)^{k_{1}}\left[k_{1}\right]^{-1 / 2}\left(C^{k_{1}}\left(\mathbf{R}_{10}\right) C^{k_{1}}\left(\mathbf{R}_{20}\right)\right)} \\
\quad \times \delta_{k_{1} k_{2}}=(-1)^{k_{1}}\left[k_{1}\right]^{-1 / 2} \delta_{k_{1} k_{2}} P_{k_{1}}\left(\cos \theta_{12}\right) .
\end{gathered}
$$

Для типичных лигандов с валентной $p$-оболочкой $l_{0}=1$, $l_{0}^{\prime}=0$ мы приходим к простой угловой зависимости:

$$
\begin{aligned}
I\left(b_{1} b_{2} 00\right)= & \delta_{b_{1} b_{2}}\left(J\left(b_{1} b_{1} 000\right)-\frac{1}{\sqrt{3}} J\left(b_{1} b_{1} 110\right) \cos \theta_{12}\right. \\
& \left.+\frac{1}{\sqrt{5}} J\left(b_{1} b_{1} 220\right) \frac{3 \cos ^{2} \theta_{12}-1}{2}\right) \\
= & \delta_{b_{1} b_{2}}\left[\alpha\left(b_{1}\right)+\beta\left(b_{1}\right) \cos \theta_{12}+\gamma\left(b_{1}\right) \cos ^{2} \theta_{12}\right],
\end{aligned}
$$

где первое и третье слагаемые определяют вклад внутриконфигурационных эффектов, а второе - вклад межконфигурационных $n p-n^{\prime} s$-эффектов.

Гамильтониан обменного взаимодействия двух $n d$-ионов с электронными конфигурациями $t_{2 g}^{n_{1}} e_{g}^{n_{2}}$, 
типичными для сильного кристаллического поля, имеет достаточно сложный вид $[4,5,9]$ :

$$
\begin{aligned}
\hat{V}_{\mathrm{ex}}(1,2)= & \sum_{a \gamma_{1}, \gamma_{2}} I^{*}\left(f_{1} \Gamma_{1} \Gamma_{1}^{\prime} f_{2} \Gamma_{2} \Gamma_{2}^{\prime} \mid \gamma_{1} \gamma_{2} \gamma v\right) \\
& \times\left[\hat{W}^{a \gamma_{1}}\left(\Gamma_{1} \Gamma_{1}^{\prime}\right) \times \hat{W}^{a \gamma_{2}}\left(\Gamma_{2} \Gamma_{2}^{\prime}\right)\right]_{v}^{\gamma},
\end{aligned}
$$

где

$$
\left[\hat{W}^{a \gamma_{1}} \times \hat{W}^{a \gamma_{2}}\right]_{v}^{\gamma}=\sum_{\alpha v_{1} \nu_{2}}\left[\begin{array}{lll}
\gamma_{1} & \gamma_{2} & \gamma \\
\nu_{1} & \nu_{2} & v
\end{array}\right](-1)^{\alpha} \hat{W}_{\alpha v_{1}}^{a \gamma_{1}} \hat{W}_{-\alpha \nu_{2}}^{a \gamma_{2}}
$$

есть тензорное произведение двойных тензорных операторов, являющееся скаляром в спиновом пространстве и тензором ранга $\gamma$ в орбитальном пространстве. Отметим, что волновые функции и тензорные операторы заданы в (11) в локальных системах координат. Для янтеллеровских ионов $E$-типа $\left(\mathrm{Cu}^{2+}, \mathrm{Mn}^{3+}\right)$ можно использовать псевдоспиновый формализм с состояниями $|E 2\rangle$ и $|E 0\rangle$, приписываемыми $|+1 / 2\rangle$ и $|-1 / 2\rangle$ состояниям псевдоспина $\tau=1 / 2$. Орбитальные части операторов $\hat{W}^{a E}$ могут быть заменены следующим образом:

$$
\hat{W}_{0}^{a A_{1}} \Rightarrow \hat{1} ; \quad \hat{W}_{0}^{a E} \Rightarrow \hat{\tau}_{z} ; \quad \hat{W}_{2}^{a E} \Rightarrow \hat{\tau}_{x} .
$$

После такого преобразования гамильтониан (1) дает обобщение псевдоспинового гамильтониана КугеляХомского [10].

С учетом кинетического и потенциального вкладов обменные параметры могут быть представлены в виде:

$$
\begin{aligned}
& I\left(f_{1} \Gamma_{1} \Gamma_{1}^{\prime} f_{2} \Gamma_{2} \Gamma_{2}^{\prime} \mid \gamma_{1} \gamma_{2} \gamma v\right)=\sum\left[\gamma_{1} \gamma_{2}\right]^{\frac{1}{2}}\left[\begin{array}{lll}
\gamma_{1} & \gamma_{2} & \gamma \\
v_{1} & v_{2} & v
\end{array}\right]^{*} \\
& \times(-1)^{j\left(\Gamma_{1}\right)-\mu_{1}}\left\langle\begin{array}{ccc}
\Gamma_{1} & \gamma_{1} & \Gamma_{1}^{\prime} \\
-\mu_{1} & \nu_{1} & \mu_{1}^{\prime}
\end{array}\right\rangle(-1)^{j\left(\Gamma_{2}\right)-\mu_{2}}\left\langle\begin{array}{ccc}
\Gamma_{2} & \gamma_{2} & \Gamma_{2}^{\prime} \\
-\mu_{2} & v_{2} & \mu_{2}^{\prime}
\end{array}\right\rangle \\
& \times\left\{\frac{t\left(f_{1} \Gamma_{1} \mu_{1} \mid f_{2} \Gamma_{2}^{\prime} \mu_{2}^{\prime}\right) t\left(f_{2} \Gamma_{2} \mu_{2} \mid f_{1} \Gamma_{1}^{\prime} \mu_{1}^{\prime}\right)}{U}\right. \\
& \left.-\frac{1}{2} J\left(f_{1} \Gamma_{1} \mu_{1} f_{2} \Gamma_{2} \mu_{2} \mid f_{2} \Gamma_{2}^{\prime} \mu_{2}^{\prime} f_{1} \Gamma_{1}^{\prime} \mu_{1}^{\prime}\right)\right\} .
\end{aligned}
$$

Легко видеть, что при учете только внутриконфигурационных эффектов $\left(\Gamma_{1}=\Gamma_{1}^{\prime}, \Gamma_{2}=\Gamma_{2}^{\prime}\right)$ обменные параметры обращаются в нуль, если $j\left(\Gamma_{1}\right)+j\left(\Gamma_{2}\right)$ нечетно.

\section{3. Критический анализ обобщенного (сверх)обменного гамильтониана}

Несмотря на сложный вид обобщенного гамильтониана (сверх)обменного взаимодействия, он получен в рамках хотя и стандартных, но достаточно грубых приближений. Так, при выводе обобщенных гамильтонианов (1) и (2) мы ограничились учетом только одночастичного переноса в частично заполненные подоболочки. Как показано в работах [4,11] учет межэлектронных взаимодействий в кинетическом обмене приводит к заметной перенормировке величин обменных интегралов, особенно важной с учетом частичной компенсации вкладов кинетического и потенциального обмена. Не приводя деталей, ограничимся результатами расчета обменных интегралов для основных и возбужденных состояний ионов $\mathrm{Mn}^{2+}$ в $\mathrm{KMnF}_{3}: I\left({ }^{6} A_{1}-{ }^{6} A_{1}\right)=2.5 \mathrm{~K}$ (экспериментальное значение $3.5 \mathrm{~K}) ; I\left({ }^{4} A_{1}-{ }^{6} A_{1}\right)=1.8 \mathrm{~K} ; I\left({ }^{4} A_{1}-{ }^{4} A_{1}\right)=1.5 \mathrm{~K}$. Для параметра $K$, определяющего амплитуду вероятности обменного переноса энергии возбуждения ${ }^{4} A_{1}$ с одного иона на другой, а также и энергетическую структуру уровней возбужденного $\left({ }^{4} A_{1}-{ }^{6} A_{1}\right)$-состояния пары

$$
\begin{aligned}
\Delta E & =\frac{1}{2} I\left({ }^{4} A_{1}-{ }^{6} A_{1}\right) \\
& \times\left[S(S+1)-S_{1}\left(S_{1}+1\right)-S_{2}\left(S_{2}+1\right)\right] \pm K S(S+1),
\end{aligned}
$$

где $S-$ полный спин пары, получено значение $K=1.4 \mathrm{~K}$. Кстати, при этом для величины $I^{\prime}=I\left({ }^{4} A_{1}-{ }^{6} A_{1}\right)+K$ в паре $\mathrm{Mn}^{2+}-\mathrm{Mn}^{2+}$ в изоструктурном $\mathrm{KMnF}_{3}$ диамагнетике $\mathrm{RbMnF}_{3}$ получено значение $I^{\prime}=3.2 \mathrm{~K}$, близкое к экспериментальному значению этой величины $(3.5 \mathrm{~K}[12])$. Мы видим, что даже для орбитально невырожденных состояний обменные параметры в основном и возбужденном состоянии могут заметно отличаться.

Учет переноса электрона в пустые подоболочки особенно важен для таких ионов как $\mathrm{Cr}^{3+}$ с конфигурацией $t_{2 g}^{3}$. Соответствующие вклады в обменные интегралы для пар $\mathrm{Cr}^{3+}-\mathrm{Cr}^{3+}$ и $\mathrm{Fe}^{3+}-\mathrm{Cr}^{3+}$ имеют вид

$$
\begin{gathered}
\Delta I_{\mathrm{CrCr}}=-\frac{\Delta E(35)}{6 U} \frac{t_{\sigma \pi}^{2}}{U} \sin ^{2} \theta, \\
\Delta I_{\mathrm{FeCr}}=-\frac{\Delta E(35)}{10 U}\left[\frac{\left(t_{s s}+t_{\sigma \sigma} \cos \theta\right)^{2}}{U}+\frac{t_{\sigma \pi}^{2}}{U} \sin ^{2} \theta\right],
\end{gathered}
$$

где $t_{s s}, t_{\sigma \sigma}, t_{\sigma \pi}$ - интегралы переноса электрона с участием соответствующих связей, $\Delta E(35)$ - разница энергий низкоспинового ${ }^{3} E_{g}$ - и высокоспинового ${ }^{5} E_{g}$-термов конфигурации $t_{2 g}^{3} e_{g}$, соответствующей иону $\mathrm{Cr}^{2+}$. Очевидно, что оба вклада имеют ферромагнитный знак. В результате суммарный кинетический вклад в обменный интеграл $I\left(\mathrm{Cr}^{3+} \mathrm{Cr}^{3+}\right)$ может даже изменить знак при некотором критическом значении угла сверхобменной связи $\theta=\theta_{c r}$.

Везде выше кинетический вклад в обменные параметры учитывался в простейшем приближении учета „средней“ энергии переноса $U$. Это популярное приближение достаточно трудно обосновать даже для обмена в основных состояниях, тогда как для возбужденных состояний оно может привести к ошибочным оценкам не только величины, но и знака обменных параметров.

Обобщенные гамильтонианы (1) и (11) формально применимы только для анализа обменных взаимодействий в паре отдельных ионов или катион-анионных кластеров типа октаэдрических комплексов $3 d$-элементов 
с конфигурацией $t_{2 g}^{n_{1}} e_{g}^{n_{2}}$. Другими словами, их можно использовать для описания обменных эффектов либо в пределах одной и той же конфигурации типа $3 d^{n}$, $t_{2 g}^{n_{1}} e_{g}^{n_{2}}$ или нескольких конфигураций, но отличающихся всего лишь заполнением $t_{2 g}$ - и $e_{g}$-подоболочек. В частности, это относится к описанию роли обменных взаимодействий для запрещенных внутрицентровых $d-d$-переходов (crystal-field transitions), но не переходов c переносом заряда. Конечные (возбужденные) состояния для разрешенных и запрещенных внутрицентровых $p-d$-переходов с переносом заряда в комплексах переходных элементов соответствуют конфигурациям c p-дыркой, локализованной на ближайших лигандах, что приводит к существенному изменению характера обменного взаимодействия с окружением, прежде всего благодаря „включению“ сильного $p-d$-обмена. Межцентровые $d-d$-переходы с переносом заряда типа $d^{n}-d^{n} \rightarrow d^{n+1}-d^{n-1}$ приводят к изменению электронных конфигураций двух центров. Отметим, что именно переходы с переносом заряда формируют полосу фундаментального поглощения в подавляющем числе соединений типа окислов $3 d$-элементов [13-16].

Выше мы не рассматривали эффектов взаимодействия с решеткой, молчаливо предполагая применимость модели „замороженной“ (quenched) решетки как при расчетах обменного взаимодействия, так и при описании возбужденных электронных состояний. Роль электрон-решеточного взаимодействия не ограничивается только снятием запрета с внутрицентровых $d-d$-переходов. Эффекты электрон-решеточной поляризации приводят к релаксации быстрого „франккондоновского“ оптического возбуждения с возможным формированием устойчивых электрон-дырочных (ЕН) пар. Особенно важным этот эффект является для возбуждений с переносом заряда, представляющих собой „гигантскую“ зарядовую флуктуацию. Так, минимальная энергия, необходимая для рождения ЕН-пары путем прямого франк-кондоновского оптического перехода с переносом заряда в родительских купратах, то есть оптическая щель, составляет $E_{\text {gap }}^{\text {opt }} \approx 1.5-2 \mathrm{eV}$. Эффекты электрон-решеточной релаксации приводят либо к распаду ЕН-пары (ЕН-рекомбинации), либо к образованию метастабильного ЕН-димера, устойчивость которого поддерживается локальной деформацией решетки и электронной поляризацией окружения. Энергия метастабильного ЕН-димера определяет „адиабатическую“, или „термическую“, щель с переносом заряда $E_{\text {gap }}^{\text {th }}$, которая может существенно отличаться от оптической щели. В родительских купратах типа $\mathrm{La}_{2} \mathrm{CuO}_{4}$ эта щель имеет удивительно малую величину порядка 0.4 эВ [17]. Очевидно, что корректный анализ роли обменных эффектов должен включать учет эффектов электрон-решеточного взаимодействия.

На практике часто обменное взаимодействие (4) рассматривают классически, заменяя операторы спина $\hat{\mathbf{S}}$ на классические векторы $\mathbf{S}$. Очевидно, что это может приводить к ошибкам, особенно существенным для квантовых спинов $s=1 / 2$. Так, точная квантовая энергия состояний пары обменносвязанных спинов $S_{1}$ и $S_{2}$ с полным спином пары $S_{12}\left(\left|S_{1}-S_{2}\right| \leq S_{12} \leq S_{1}+S_{2}\right)$ равна

$$
E\left(S_{12}\right)=\frac{I_{12}}{2}\left[S_{12}\left(S_{12}+1\right)-S_{1}\left(S_{1}+1\right)-S_{2}\left(S_{2}+1\right)\right],
$$

что дает для состояний с максимальным и минимальным спином $E\left(S_{1}+S_{2}\right)=I_{12} S_{1} S_{2}$ и $E\left(S_{1}-S_{2}\right)=-I_{12} S_{2}$ $\times\left(S_{1}+1\right)$ (при $\left.S_{1} \leq S_{2}\right)$ соответственно, тогда как для их классических аналогов - ферро- и антиферромагнитного состояний - получаем $E(F M)=I_{12} S_{1} S_{2}$ $=E\left(S_{1}+S_{2}\right)$, но $E(A F M)=-I_{12} S_{1} S_{2} \neq E\left(S_{1}-S_{2}\right)$. Таким образом, классическая „неелевская““ антиферромагнитная аналогия для состояний с минимальным полным спином пары, строго говоря, некорректна. Так, в случае квантовых спинов $S_{1}=S_{2}=1 / 2$ квантовая энергия синглетного состояния пары $E\left(S_{1}-S_{2}=0\right)=-\frac{3}{4} I_{12}$ в три раза отличается от классической энергии антиферромагнитного состояния $E(A F M)=-\frac{1}{4} I_{12}$. Именно с этим обстоятельством связаны проблемы описания основного состояния антиферромагнетиков, хотя на практике чаще всего ограничиваются все-таки классическим неелевским описанием.

\section{3. Обменно-релятивистские эфффекты в возбужденных состояниях $3 d$-ионов в кристаллах}

Во втором приближения теории возмущений с участием обменного взаимодействия в форме обобщенного гамильтониана (11) и обычного спин-орбитального взаимодействия мы получаем ряд эффективных обменнорелятивистских взаимодействий, наиболее популярным среди которых является спин-спиновый антисимметричный обмен Дзялошинского-Мория $[18,19]$, описываемый гамильтонианом

$$
V_{D M}=\sum_{i j}\left(\mathbf{d}_{i j} \cdot\left[\mathbf{S}_{i} \times \mathbf{S}_{j}\right]\right),
$$

где $\mathbf{d}_{i j}$ - так называемый вектор Дзялошинского. Развитие теории антисимметричного обмена было стимулировано открытием нового класса магнитоупорядоченных материалов - слабых ферромагнетиков. Слабые ферромагнетики - многоподрешеточные магнетики, магнитные моменты подрешеток в которых не точно антипараллельны, а имеют, как правило, небольшой явный (overt canting) или скрытый (hidden canting) скос, приводящий к появлению суммарного магнитного момента и/или поперечному слабому антиферромагнетизму.

Кеффером [20] была феноменологически предложена и Москвиным [8] теоретически выведена для сверхобменно-связанных ионов $S$-типа очень простая и изящная формула связи вектора Дзялошинского с геометрией сверхобмена, полностью согласующаяся с правилами Мория [19]:

$$
\mathbf{d}_{i j}=d_{i j}(\theta)\left[\mathbf{r}_{i} \times \mathbf{r}_{j}\right],
$$


где

$$
d_{i j}(\theta)=d_{1}\left(R_{i}, R_{j}\right)+d_{2}\left(R_{i}, R_{j}\right) \cos \theta_{i j},
$$

$\mathbf{r}_{i, j}$ - единичные векторы в направлении связей анион-катион $\mathrm{O}-M_{i, j}, \theta_{i j}-$ угол сверхобменной связи $M_{i}-\mathrm{O}-M_{j}$, а параметры $d_{1,2}\left(R_{i}, R_{j}\right)$ зависят как от типа ионов $M_{1}$ и $M_{2}$, так и от расстояний катион-анион. Знак скалярного параметра $d_{i j}(\theta)$ называют знаком вектора Дзялошинского. В условиях одинаковой геометрии сверхобмена как величина, так и знак вектора Дзялошинского определяются электронной структурой ионов $M_{1}$ и $M_{2}$ [21-25]. Подчеркнем, что рассматриваемая форма вектора Дзялошинского справедлива только для магнитных ионов $S$-типа, т.е. ионов с орбитально невырожденным основным состоянием, в частности, $3 d$-ионов с наполовину заполненными подоболочками $\left(3 d^{5}, t_{2 g}^{3}\right.$, $\left.t_{2 g}^{3} e_{g}^{2}, t_{2 g}^{6} e_{g}^{2}\right)$.

\section{1. Взаимодействия спин-чужая орбита}

Обменно-релятивистские эффекты второго приближения теории возмущений с участием обменного взаимодействия в форме обобщенного гамильтониана (1), (11) и обычного спин-орбитального взаимодействия приводят к появлению нового типа эффективных спин-орбитальных взаимодействий - взаимодействию спин-чужая орбита (spin-other orbit) $\hat{V}_{\text {SoO }}$, актуального для основных или возбужденных состояний с незамороженным орбитальным моментом типа $T_{1^{-}}, T_{2}$-состояний $d$-ионов в высокосимметричном окружении. Билинейная часть $\hat{V}_{\text {SoO }}$ для взаимодействия таких состояний с окружающими ионами $S$-типа включает изотропный и анизотропный симметричные вклады, а также анизотропный антисимметричный вклад - спин-орбитальный аналог взаимодействия Дзялошинского-Мория [26]:

$$
\begin{aligned}
\hat{V}_{\mathrm{SoO}}= & \sum_{m>n} \lambda_{m n}^{(0)}\left(\mathbf{L}_{m} \cdot \mathbf{S}_{n}\right)+\sum_{m>n}\left(\lambda_{m n} \cdot\left[\mathbf{L}_{m} \times \mathbf{S}_{n}\right]\right) \\
& +\sum_{m>n}\left(\mathbf{L}_{m} \overleftrightarrow{\boldsymbol{\lambda}}_{m n} \mathbf{S}_{n}\right) .
\end{aligned}
$$

Интересно, что вклад в билинейное взаимодействие $\hat{V}_{\mathrm{SoO}}$ вносит как спин-зависимый обмен (слагаемое с $a=1$ в обобщенном гамильтониане обмена (11), так и спиннезависимый, чисто орбитальный обмен (слагаемое с $a=0$ в гамильтониане (11)). Однако спин-зависимый обмен приводит к появлению дополнительных нелинейных, квадратичных по спину слагаемых, вклад которых может быть учтен формальной заменой линейного спинового оператора $\mathbf{S}_{n}$ в (18) на нелинейный оператор $\mathbf{S}_{m n}$

$$
\begin{aligned}
\hat{S}_{q}(m n) & =\hat{S}_{q}(n)+\gamma\left[\hat{V}^{2}(S(m)) \times S^{1}(n)\right]_{q}^{1} \\
& =\hat{S}_{q}(n)+\gamma \sum_{q_{1}, q_{2}}\left[\begin{array}{rrr}
2 & 1 & 1 \\
q_{1} & q_{2} & q
\end{array}\right] \hat{V}_{q_{1}}^{2}(S(m)) S_{q_{2}}(n),
\end{aligned}
$$

где $V_{q}^{2}(S)$ - спиновый неприводимый тензорный оператор ранга 2. В частности,

$$
\hat{V}_{0}^{2}(S)=2\left[\frac{(2 S-2)}{(2 S+3)}\right]^{1 / 2}\left(3 \hat{S}_{z}^{2}-S(S+1)\right) .
$$

Коэффициент $\gamma$ в (19) рассчитывается для конкретных термов.

Изотропная часть $V_{s o}^{e x}$ в общем случае может быть представлена в виде

$$
\begin{aligned}
V_{s o}^{e x}= & \sum_{m n} \lambda(m n)(\mathbf{L}(m) \cdot \mathbf{S}(n)) \\
& +\sum_{m \neq n} \lambda^{\prime}(m n)(\mathbf{L}(m) \cdot \mathbf{S}(m))(\mathbf{S}(m) \cdot \mathbf{S}(n)) .
\end{aligned}
$$

Аналогично вектору Дзялошинского, для оценки параметров взаимодействия спин-чужая орбита можно воспользоваться простым соотношением

$$
\lambda(m n) \approx \frac{\lambda^{\prime} I^{\prime}}{\Delta E_{S \Gamma}}
$$

где $\lambda^{\prime}$ и $I^{\prime}-$ константа спин-орбиты для $T_{1}$-, $T_{2}$-состояний и параметр недиагонального обмена, $\Delta E_{S \Gamma}-$ некоторая энергия возбуждения. Простые оценки показывают, что благодаря $\hat{V}_{\mathrm{SoO}}$ эффективные магнитные поля, действующие на орбитальные $T_{1}$-, $T_{2}$-состояния, например ионов $\mathrm{Fe}^{3+}$ в ферритах, могут достигать величин порядка $100 T$ и более.

\section{2. Обменно-релятивистские взаимодействия и циркулярная магнитооптика}

Наиболее ярко спин-орбитальные взаимодействия спин-чужая орбита проявляются в циркулярных магнитооптических эффектах Фарадея и Керра в системах типа ферритов с магнитными ионами $S$-типа. Дело в том, что эти эффекты определяются орбитальными магнитными полями в возбужденных состояниях, которые в системах с ионами $S$-типа в отсутствие внешнего поля могут быть индуцированы только взаимодействиями спин-чужая орбита.

Циркулярная магнитооптика (циркулярное двупреломление и дихроизм) определяется аксиальным вектором гирации $\mathbf{g}=\sum_{i} \boldsymbol{\alpha}(i)$. Так, фарадеевское вращение $\Theta_{F}$ в некубических кристаллах может быть записано в виде

$$
\Theta_{F}=A(\mathbf{g} \cdot \mathbf{n}),
$$

где $\mathbf{n}$ - единичный вектор распространения света, $A-$ фактор, зависящий от направления распространения света и его поляризации, а также главных значений коэффициента преломления. Вектор гирации имеет те же свойства симметрии, что и магнитный момент, или вектор ферромагнетизма $\mathbf{F}$, что позволяет представить 
его в виде

$$
\mathbf{g}=\overleftrightarrow{\alpha} \mathbf{F}+\overleftrightarrow{\gamma} \mathbf{H}_{\mathrm{ext}}
$$

т. е. суммы ферромагнитного и диамагнитного вкладов соответственно. Однако в слабых ферромагнетиках некоторые „ортогональные“ компоненты векторов ферромагнетизма $\mathbf{F}$ и антиферромагнетизма $\mathbf{G}$ имеют одинаковые свойства симметрии, что указывает на существование в них специфического „антиферромагнитного“ вклада в вектор гирации

$$
\Delta \mathbf{g}=\overleftrightarrow{\boldsymbol{\beta}} \mathbf{G}
$$

а значит, в магнитооптические эффекты Фарадея и Керра. Так, в орторомбических слабых ферромагнетиках - ортоферритах типа $\mathrm{YFeO}_{3}$ - отличны от нуля компоненты $\beta_{x z}$ и $\beta_{z x}$ тензора $\overleftrightarrow{\boldsymbol{\beta}}$. Соотношение $F \ll G$, типичное для слабых ферромагнетиков, указывает на, возможно, существенный антиферромагнитный вклад в вектор гирации даже при относительно малой величине компонент тензора $\overleftrightarrow{\boldsymbol{\beta}}$. С учетом внешнего магнитного поля, локального и нелокального спин-орбитального взаимодействия представим вклад в вектор гирации разрешенного перехода ${ }^{6} A_{1 g}-{ }^{6} T_{1 u}$ в октаэдрическом комплексе $\mathrm{FeO}_{6}$ как [26]:

$$
\begin{aligned}
\mathbf{g}= & \left(\frac{n_{0}^{2}+2}{3}\right)^{2} \frac{2 \pi e^{2} f_{A T}}{m \omega_{0}} \frac{\partial F_{1}\left(\omega, \omega_{0}\right)}{\partial \omega_{0}} \\
& \times\left(-N \beta_{e} \mathbf{H}_{\mathrm{ext}}+\lambda_{\mathrm{eff}} \sum_{m}\left\langle\mathbf{S}_{m}\right\rangle+\sum_{m>n} \lambda_{m n}^{(0)}\left\langle\mathbf{S}_{n}\right\rangle\right. \\
& \left.-\sum_{m>n}\left[\lambda_{m n} \times\left\langle\mathbf{S}_{n}\right\rangle\right]+\sum_{m>n} \overleftrightarrow{\lambda}_{m n}\left\langle\mathbf{S}_{n}\right\rangle\right)
\end{aligned}
$$

где $N$ - число кластеров $\mathrm{FeO}_{6}$ в единице объема, $f_{A T}$ и $\hbar \omega_{0}-$ сила осциллятора и энергия ${ }^{6} A_{1 g}-{ }^{6} T_{1 u}$-перехода, $F_{1}\left(\omega, \omega_{0}\right)$ - дисперсионный фактор. Очевидно, что нетривиальный антиферромагнитный вклад в вектор гирации определяется нелокальными анизотропными спинорбитальными взаимодействиями, то есть взаимодействиями спин-чужая орбита, причем спин-орбитальный аналог взаимодействия Дзялошинского-Мория дает вклад в антисимметричную часть тензора $\overleftrightarrow{\boldsymbol{\beta}}$, а симметричное анизотропное спин-орбитальное взаимодействие - в симметричную часть тензора $\overleftrightarrow{\boldsymbol{\beta}}$. Экспериментальные исследования эффекта Фарадея в слабом ферромагнетике $\mathrm{YFeO}_{3}$ [27], прежде всего, зависимости $\Theta_{F}\left(\mathbf{H}_{\text {ext }}\right)$ позволили оценить все вклады в вектор гирации $(\lambda=0.6328 \mu \mathrm{m})$ :

$$
\begin{gathered}
\alpha_{z z} F_{z}=(0.95 \pm 0.55) \cdot 10^{-3} ; \\
\left.\beta_{z x} G_{x}=(3.15 \pm 0.55)\right) \cdot 10^{-3} ; \\
\alpha_{x x} F_{x}=(0.2 \pm 0.7) \cdot 10^{-3} ; \\
\left.\beta_{x z} G_{z}=(-2.1 \pm 1.0)\right) \cdot 10^{-3} ; \\
\gamma_{z z} \approx \gamma_{x x}=(-1.1 \pm 2.8) \cdot 10^{-6} \mathrm{kO} e^{-1} .
\end{gathered}
$$

Несмотря на относительно большие ошибки, можно сделать однозначный вывод о большом, если не определя- ющем, антисимметричном антиферромагнитном вкладе, что можно рассматривать как экспериментальное подтверждение важной роли обменно-релятивистского взаимодействия спин-чужая орбита, прежде всего, спинорбитального аналога взаимодействия ДзялошинскогоМория.

В ряде редкоземельных ортоферритов $R \mathrm{FeO}_{3}(R=\mathrm{Nd}$, $\mathrm{Sm}, \mathrm{Tb}, \mathrm{Ho}, \mathrm{Er}, \mathrm{Tm}, \mathrm{Yb})$ наблюдается явление спонтанной спин-переориентации из высокотемпературной фазы $\Gamma_{4}\left(G_{x}, F_{z}\right)$ в низкотемпературную фазу $\Gamma_{2}\left(F_{x}, G_{z}\right)$, при которой вектор ферромагнетизма (магнитный момент) поворачивается от $c$-оси к $a$-оси. Это дает замечательную возможность исследования различных компонент вектора гирации

$$
\begin{array}{cc}
g_{x}=\alpha_{x x} F_{x}+\beta_{x z} G_{z}=\alpha_{x x} F_{x}+\beta_{z x}^{a}-\beta_{z x}^{s} & \left(\Gamma_{2}-\text { фаза }\right) \\
g_{z}=\alpha_{z z} F_{z}+\beta_{z x} G_{x}=\alpha_{z z} F_{z}+\beta_{z x}^{a}+\beta_{z x}^{s} & \left(\Gamma_{4}-\text { фаза }\right) \\
\left(\text { при } G_{x}=+1, \quad G_{z}=-1\right) .
\end{array}
$$

Теоретическая обработка магнитооптических спектров $\mathrm{TmFeO}_{3}$ в области $p-d$ переходов с переносом заряда в фазах $\Gamma_{2}$ и $\Gamma_{4}[26]$ дает возможность оценить величину эффективных орбитальных магнитных полей для ${ }^{6} T_{1 u}$-термов конфигураций с $p-d$-переносом заряда в октакомплексах $\mathrm{FeO}_{6}$. Так, для ${ }^{6} T_{1 u}$-терма с энергией 3.15 эВ орбитальное поле составляет $130 \mathrm{~T}$ в фазе $\Gamma_{4}$ и $90 \mathrm{~T}$ в фазе $\Gamma_{2}$, что предполагает существенный симметричный антиферромагнитный вклад в вектор гирации и, скорее всего, ведущий антисимметричный вклад.

Интересно, что для тригональных слабых ферромагнетиков $\alpha-\mathrm{Fe}_{2} \mathrm{O}_{3}, \mathrm{FeBO}_{3}$ антиферромагнитный вклад в вектор гирации полностью определяется только антисимметричным спин-орбитальным взаимодействием.

\section{4. Заключение}

Использование простейших спиновых гамильтонианов типа Гейзенберга или Дзялошинского-Мория, традиционных для основных орбитально-невырожденных состояний, не позволяет корректно описать особенности обменных и обменно-релятивистских взаимодействий для возбужденных состояний $3 d$ - и $4 f$-ионов в кристаллах. Нами дан критический анализ обобщенного гамильтониана обменного и сверхобменного взаимодействий, который в рамках единого подхода позволяет учесть эффекты орбитального (квази)вырождения, обменный механизм переноса возбуждения. Вместе с тем, стандартные приближения, заложенные в основу расчета кинетического вклада в обменные параметры, в частности, пренебрежение ролью двухчастичных корреляционных взаимодействий, использование средней энергии перено$\mathrm{ca}$, независящей от типа начального и конечного состояний, пренебрежение переносом в „пустые“ подоболочки особенно критичны для возбужденных состояний и пока ставят под сомнение возможность надежных оценок 
величины и даже знака соответствующих обменных параметров.

Рассмотрен новый механизм обменно-релятивистских взаимодействий „спин-чужая орбита““, в частности, спин-орбитальный аналог взаимодействия Дзялошинского-Мория и его проявление в циркулярной магнитооптике слабых ферромагнетиков. Орбитальные магнитные поля, индуцируемые этим взаимодействием в слабых ферромагнетиках, могут существенно превышать поля Дзялошинского и достигать величин порядка 100 Т для ${ }^{6} T_{1 u}$-термов конфигураций с переносом заряда в октакомплексах $\mathrm{FeO}_{6}$.

\section{Список литературы}

[1] В.В. Дружинин, А.С. Москвин. Физика металлов и металловедение 26, 415 (1968).

[2] P.M. Levy. Phys. Rev. 177, 509 (1969);[G.M. Copland, P.M. Levy. Phys. Rev. B 1, 3043 (1970)].

[3] I. Veltrusky. Czech. J. Phys. 25, 101 (1975).

[4] А.С. Москвин. Антисимметричный обмен и магнитная анизотропия в слабых ферромагнетиках. Дисс. доктора физ.-мат. наук, Урал. гос. университет, Свердловск (1983); http://www.dissercat.com/content/antisimmetrichnyi-obmen-imagnitnaya-anizotropiya-v-slabykh-ferromagnetikakh.

[5] А.С. Москвин. Атомы в кристаллах. / Изд-во Урал. ун-та, Екатеринбург (2018) 399 с.; http://elar.urfu.ru/handle/10995/65226

[6] А.С. Москвин, В.В. Дружинин. Оптика и спектроскопия 29, 899 (1970).

[7] A.S. Moskvin, S.-L. Drechsler. Phys. Rev. B 78, 024102 (2008); [Europhys. Letters 81, 57004 (2008)].

[8] А.С. Москвин. ФТТ 12, 3209, 1970.

[9] А.А. Сидоров, А.С. Москвин, В.В. Попков. ФТТ 18, 3005 (1976).

[10] К.И. Кугель, Д.И. Хомский. УФН 136, 621 (1982).

[11] А.С. Москвин, А.С. Лукьянов. ФТТ 19, 1975 (1977).

[12] J. Fergusson, H.J. Guggenheim, Y. Tanabe. J. Phys. Soc. Jpn. 21, 692 (1966)

[13] A.S. Moskvin, J. Malek, M. Knupfer, R. Neudert, J. Fink, R. Hayn, S.-L. Drechsler, N. Motoyama, H. Eisaki, S. Uchida. Phys. Rev. Lett. 91, 037001 (2003).

[14] A.S. Moskvin, R.V. Pisarev. Физика низких температур 36 6, 613 (2010).

[15] A.S. Moskvin, A.A. Makhnev, L.V. Nomerovannaya, N.N. Loshkareva, A.M. Balbashov. Phys. Rev. B 82, 035106 (2010).

[16] V.I. Sokolov, V.A. Pustovarov, V.N. Churmanov, V.Yu. Ivanov, N.B. Gruzdev, P.S. Sokolov, A.N. Baranov, A.S. Moskvin. Phys. Rev. B 86, 115128 (2012).

[17] A.S. Moskvin. Phys. Rev. B 84, 075116 (2011).

[18] И.Е. Дзялошинский. ЖЭТФ 32, 1547 (1957); [I. Dzyaloshinsky. J. Phys. Chem. Solids 4, 241 (1958)].

[19] T. Moriya. Phys. Rev. Lett. 4, 228 (1960); [Phys. Rev. 120, 91 (1960)].

[20] F. Keffer. Phys. Rev. 126, 896 (1962).

[21] А.С. Москвин, И.Г. Бострем. ФТТ 19, 1616 (1977).

[22] А.С. Москвин. ФТТ 32, 1644 (1990).

[23] A.S. Moskvin. ЖЭТФ 131, 1048 (2007).

[24] A.S. Moskvin. Phys. Rev. B 75, 054505 (2007).
[25] A.S. Moskvin. JMMM, 400, 117 (2016); [ibid, 463, 50 (2018)].

[26] Е.А. Ганьшина, А.В. Зенков, Г.С. Кринчик, А.С. Москвин, А.Ю. Трифонов. ФТТ 33, 1122 (1991).

[27] А.В. Зенков, Б.Б. Кричевцов, А.С. Москвин, К.М. Мукимов, Р.В. Писарев, М.М. Рувинштейн. ЖЭТФ 96, 1397 (1989).

Редактор Ю.Э. Китаев 